\title{
TRATAMIENTO DE LA HIPERTROFIA BENIGNA DE PRÓSTATA CON EL LÁSER REVOLIX ${ }^{\circledR}$
}

\author{
Stefano Mattioli', Rubén Muñoz, Ricard Recasens, Carlos Berbegal, Jordi Cortada², José M. Urmeneta ${ }^{3}$ \\ y Heinrich Teichmann ${ }^{4}$.
}

IInstituto Clinico San Ambrogio. Milan. Italia.

${ }^{2}$ Hospital Montserrat. Lleida. España.

${ }^{3}$ Clínica del Remei. Barcelona. España.

${ }^{4}$ Lisa. Katlenburg-Lindau. Germany.

\begin{abstract}
Resumen.- OBJETIVO: El tratamiento de la hipertrofia prostática benigna (HBP) mediante el láser Revolix $\circledast$ es eficaz y seguro. Su versatilidad permite la utilización de dos tipos de técnicas: la vaporesección y la vaporización. Su target cromóforo es el agua, como sucede con el láser Holmiun, con la diferencia que se puede utilizar a modo continuo y a modo pulsado. La onda continua permite realizar cortes precisos y excelente hemostasia sin afectar a los tejidos vecinos 10.2 mms de penetración debajo del cortel con una excelente visión del campo operatorio. Presentamos nuestra experiencia después de 3 años de tratamiento.
\end{abstract}

MÉTODOS: El láser Revolix® (Thulium) tiene una longitud de onda de $2013 \mathrm{~nm}$. Se utilizan dos tipos de fibras para tratar la HBP que alcanzan hasta una potencia de 70 vatios. Una fibra frontal para la vaporesección y una fibra con emisión de energía láser lateral para la vaporización. Ambas fibras pueden combinarse para tratar la hipertrofia prostática.
Rubén Dario Muñoz Montastruc

Hospital Montserrat

Apartado 643

25080 Lleida. (España).

rudamu@hotmail.fr
Desde finales del 2004 hasta marzo del 2008 se trataron 200 pacientes por HBP. Los tamaños de las próstatas oscilaron entre 20 y 120 grs (promedio $45 \mathrm{grs}$ ). Se realizó vaporización de la próstata en 99 pacientes, con tamaño menor de 35 gramos, y se hizo vaporesección en 101 pacientes, cuyas próstatas eran mayores de 35 gramos. El tiempo medio de hospitalización fue de 24 horas (rango 1-4 dias). El tiempo medio de permanencia de la sonda de Foley tras el tratamiento con láser fue de 16 horas (rango 12-72 horas). La efectividad del tratamiento de la próstata mediante el láser Revolix® fue evaluada principalmente con la flujometria, midiendo el residuo postmiccional y evaluando la sintomatología mediante el cuestionario IPSS.

RESULTADOS: Los resultados clínicos obtenidos tras operar a los pacientes con HBP mediante el láser Revolix $\Theta_{\text {son com- }}$ parables a los que se obtienen con la resección transuretral de próstata, la vaporización con KTP ó la enucleación con láser Holmium. Se demuestra una importante mejoría de los síntomas, con una diminución de más del 50\% del IPSS, un aumento significativo en la flujometría y un marcado descenso del residuo postmiccional. No se precisó hacer ninguna transfusión sanguinea. Hubo solamente 4 pacientes que presentaron retención aguda de orina postoperatoria, que requirieron colocación de sonda vesical durante unos días, recuperando la micción espontánea posteriormente. No hubo ningún paciente que presentara incontinencia urinaria tras el tratamiento con el láser Revolix®.

CONCLUSIONES: En nuestra experiencia, el láser Revolix $\circledast$ ha demostrado ser muy seguro, de fácil instalación, con bajo costo operativo y con una curva de aprendizaje muy corta. Su efectividad se puede poner más de manifiesto empleando la vaporesección en las próstatas voluminosas y la vaporización en las próstatas más pequeñas (menores de 35 grs). La reducción del tejido prostático es inmediata con un ligero desconfort post operatorio y un bajo índice de complicaciones. 
Palabras clave: Vaporesección. Vaporización. HBP.

Fotones. Cromóforo. Láser.

Summary.- OBJECTIVES: The treatment of benign prostatic hyperplasia (BPH) with the Revolix $\Theta$ laser is effective. Its versatility enables 2 types of techniques: vaporresection and vaporization. Its chromophore target is water, like the holmium laser, with the difference that it may be used in both a continuous and pulsed mode. The continuous wave enables a precise cut and excellent hemostasis without diffusion to the neighbor tissues $(0.2 \mathrm{~mm}$ penetration below the cut zone) with an excellent vision of the operative field. We report our experience after three years of treatment.

METHODS: Revolix ${ }^{\circledR}$ (thulium) laser has a wavelength of $2013 \mathrm{~nm}$. 2 types of fibers, which reach a potency of up to 70 watts, are used to treat BPH. One frontal fiber for vaporresection and one fiber with side laser emission for vaporization. Both fibers may be combined to treat prostatic hypertrophy. Since the end of 2004 to March 2008200 patients with BPH were treated. Prostate sizes varied from 20 to $120 \mathrm{~g}$ (mean $45 \mathrm{~g}$. 99 patients underwent prostate vaporization, with sizes below $35 \mathrm{~g}$, and 101 patients underwent vaporresection, with prostates over $35 \mathrm{~g}$. Mean hospital time was 24 hours (range 1-4 hours). Mean catheterization time after laser treatment was 16 hours (range 12-72 hours). The efficacy of the treatment was evaluated with uroflowmetry, post void residual and evaluation of the symptoms with the IPSS questionnaire.

RESULTS: The clinical results obtained after Revolix $\circledast$ laser are comparable to those obtained after transurethral resection of the prostate, KTP laser vaporization or holmium laser enucleation. We demonstrate an important improvement of symptoms, with a decrease over 50\% in the IPSS score, a significant improvement in the uroflowmetry, and a marked decrease of post void residual. No blood transfusions were required. There were only 4 patients that presented post operative acute urinary retention, requiring catheterization for a few days; all of them achieved spontaneous voiding posteriorly. No patient presented urinary incontinence after treatment with the Revolix@ laser.

CONCLUSIONS: In our experience, the Revolix ${ }^{\circledR}$ laser has demonstrated being safe, easy to install, with low operative cost, and a very short learning curve.

Its efficacy may be better demonstrated using vapor resection for big prostates and vaporization for smaller prostates (the smaller than $35 \mathrm{~g}$ ). The reduction of prostatic tissue is intermediate with a slight post operative discomfort and a low complication rate.

\section{INTRODUCCIÓN}

La utilización del láser en el tratamiento de la HBP no es una técnica nueva. En la década de los 90 ya existía un marcado interés en su utilización aunque no llegó a desarrollarse por falta de madurez tecnológica. El láser (Light Amplification by Stimulated Emission of Radiation) es en la práctica un haz estrecho de luz coherente monocromático. Este rayo luminoso es emitido y excita los electrones que liberan energia en forma de fotones. Uno de los parámetros mas importantes de la interacción del rayo láser con los tejidos es la absorción. Los fotones son absorbidos en los tejidos por moléculas particulares llamadas cromóforos. Estos son moléculas o agrupamientos de átomos responsables de la coloración de una célula y son capaces de absorber la energía de los fotones. Para el tratamiento de la próstata son utilizados dos cromóforos: el agua y la hemoglobina (oxihemoglobina) contenidos en la células. Mediante el efecto fototérmico los fotones encuentran las moléculas de cromóforos correspondientes que van a provocar una transformación energética que tiene como principio el aumento de la temperatura en el tejido elegido. En función de la duración y el aumento de la temperatura aparecen diferentes efectos desde la hipertermia hasta la evaporación tisular o la carbonización. De este efecto fototérmico surgen tres etapas importantes:

1) Etapa óptica: La energía de fotones es absorbida por las moléculas de cromóforos correspondientes provocando una vibración y luego un choque con las otras moléculas. Esto condiciona el aumento de la temperatura tisular.

2) Etapa térmica: Es la transferencia del calor por conducción. El calor se transmite desde las partículas de más a las de menos energía en función de las características propias de cada tejido o en función de los impulsos del láser.

Esta particularidad de los tejidos se llama tiempo de relajación térmica y depende del mismo tejido, su naturaleza, el volumen del tejido seleccionado y el diámetro del spot del láser. Esta característica explica el por qué de la diferencia entre un láser a modo continuo de otro a modo pulsado.

3) Etapa desnaturalización: Produce diferentes efectos dependiendo de la temperatura alcanzada:

$45^{\circ}$ Vasodilatación - Daño tisular.

$50^{\circ}$ Desaparición de la actividad enzimática.

$60^{\circ}$ Desorganización de la membrana celular y desnaturalización de las proteínas.

70을 Disminución del colágeno y de la permeabilidad de la membrana.

$80^{\circ}$ Contracción de las fibras colágenas y necrosis coagulativa.

$100^{\circ}$ Vaporización del agua y deshidratación total.

$>100^{\circ}$ Volatilización de los constituyentes orgánicos.

\section{MATERIAL Y MÉTODOS}

Hemos realizado 200 tratamientos por HBP durante un período de 40 meses (2004-2008) utilizando el láser Revolix® 70.
Keywords: Vaporresection. Vaporization. BPH. Laser. 
El Revolix® es un láser versátil, sólido y de alto rendimiento. Sus indicaciones son amplias dentro de las patologias urológicas. Se utiliza con corriente standard, sin ninguna instalación especial ni extensión de agua. La irrigación es con suero salino.

La longitud de onda es de $2013 \mathrm{~nm}$ (2 micrones) teniendo como target cromóforo el agua como sucede con el láser Holmiun (1, 2 y 3). En el espectro electromagnético se encuentra dentro de los infrarojos (invisible). Es un láser a modo continuo o pulsado y su potencia puede subir hasta 70 vatios. Las radiaciones son trasmitidas por fibras de silicio. El cristal del láser es excitado por las radiaciones emitidas por una barra de diodos láser (DPSS). Las radiaciones de los diodos lasers son dirigidas hacia un cristal que emite las radiaciones de 2 micrones $(2013 \mathrm{~nm})$. El láser de $2 \mathrm{mi}-$ crones es focalizado dentro de una fibra. El láser sale de la fibra guiado por un apropiado aplicador (resectoscopio o ureteroscopio) y es utilizado como instrumento quirúrgico. La interacción láser-tejido está basada en la fuerte absorción de radiaciones de 2 micrones por las moléculas de agua que están presentes en todos los tejidos, abstracción hecha de la coloración o de la circulación sanguínea. La penetración del rayo láser Revolix $®$ en el tejido es inferior a $0.4 \mathrm{mms}$. Esto no modifica las propiedades ópticas del tejido irradiado, con lo cual el tejido subyacente queda siempre visible al cirujano. Esta propiedad hace de este láser un instrumento quirúrgico seguro y eficaz para la cirugía de tejidos blandos.

La intensidad puede modificarse por un cambio de la potencia láser o de la distancia entre la punta de la fibra y el tejido. La dependencia de la distancia es debida a que la radiación diverge considerablemente a la salida de la fibra. Además, el efecto sobre el tejido difiere según se trabaje en medio acuoso o gaseoso. En medio acuoso el

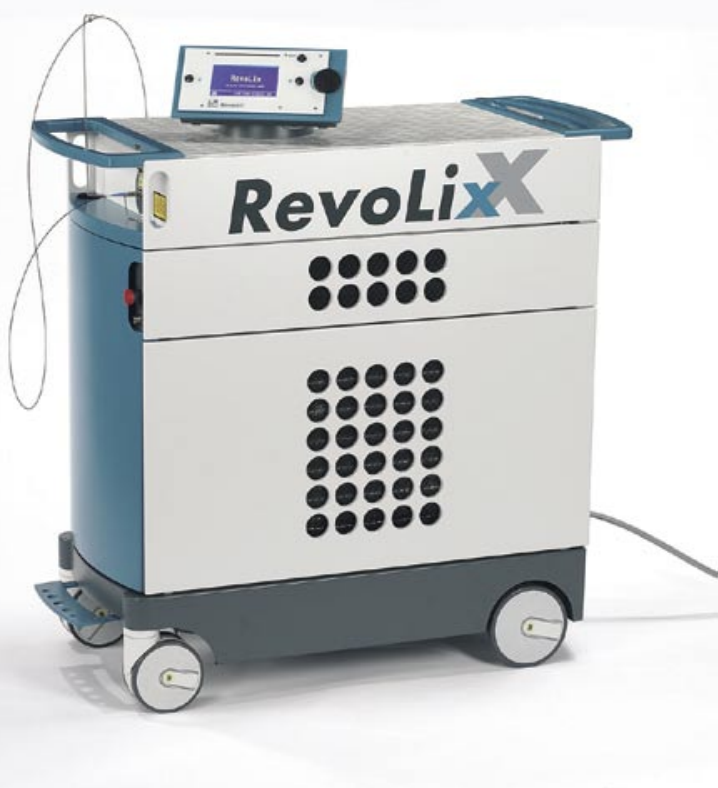

FIGURA 1. Láser Revolix 70. enfriamiento del tejido tratado es mejor, evitando así una carbonización. A baja intensidad (baja potencia o spot mas ancho) el efecto obtenido es la coagulación, pues la densidad de potencia es inferior al umbral de vaporización tisular. El efecto sobre el tejido estará limitado a un emblanquecimiento del mismo. Así mismo, una irradiación prolongada no llega a la vaporización pues el proceso de absorción de la radiación láser es combinado con la disipación del calor dentro del medio (tejido y medio acuoso). Por otra parte, el efecto de enfriamiento por conducción del calor dentro del medio compensa la acumulación de calor debido a la absorción. Con el aumento de la intensidad (potencia elevada o spot mas pequeño) la acumulación de calor dentro del tejido irradiado llega a un punto donde el agua del tejido se evapora y desaparece.

La vaporización puede controlarse cambiando la densidad (intensidad) de la potencia. Sin embargo, a un cierto punto un aumento de la densidad de la potencia no crea más aumento de la vaporización pues el tejido es protegido por los productos vaporizados que lo recubren. Si la fibra queda fija en el mismo sitio la vaporización tisular continuará, el tejido será evaporado hasta una carbonización que será fuertemente reducida en un medio acuoso si la comparamos a la cirugia en el aire ambiental.

El láser Revolix® es un láser quirúrgico utilizado en modo contacto o no, para la incisión, excisión, la vaporización y la coagulación de los tejidos blandos. El cirujano dispone de diferentes parámetros de tratamiento que le permitirá modular su técnica y el efecto del láser sobre los tejidos, entre los cuales se destacan los parámetros de potencia, el diámetro del rayo y la selección de la fibra.

1.- Parámetros de potencia: El valor de la potencia determina el volumen del tejido coagulado o vaporizado por la unidad de tiempo. Debido a la gran absorción de la radiación del láser Revolix® por el tejido, es aconsejable iniciar el tratamiento con una potencia baja.

2.- El diámetro del rayo: El rayo que sale de la fibra del aplicador diverge con un ángulo de aproximadamente $25^{\circ}$. El diámetro del rayo aumenta con la distan-

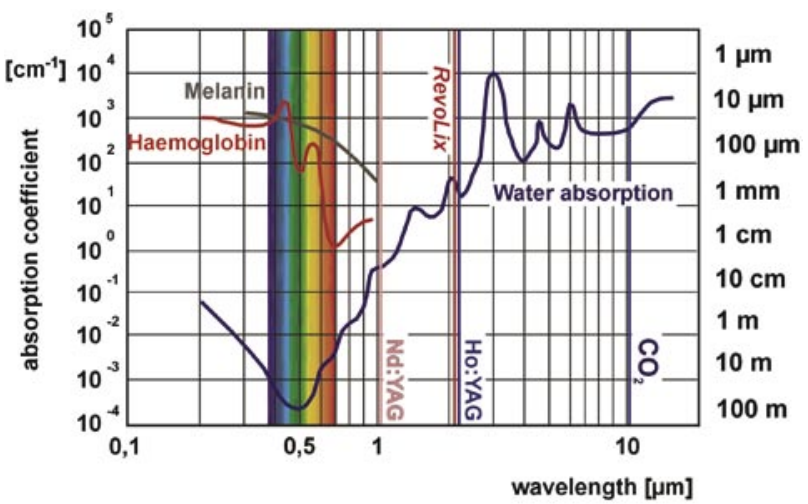

FIGURA 2. Espectro electromagnético. 
cia. La intensidad es igual a la potencia por la superficie del rayo, y es por ello que la intensidad disminuye con la distancia. La intensidad del rayo es mas fuerte a la salida de la fibra-aplicador y va disminuyendo con la distancia. La vaporación del tejido requiere una alta intensidad. Una intensidad baja se utiliza para la coagulación. En medio acuoso el rayo láser es fuertemente absorbido. En función de la potencia utilizada el tejido situado a más 1 a $4 \mathrm{mms}$ de la punta de la fibra está completamente protegido del rayo láser. Este efecto se comporta como una medida de seguridad.

3.- La selección de la fibra: Hay una gama de fibras disponibles para la utilización de este láser. Diferentes diámetros del interior de la fibra y del exterior (cladding). El diámetro interior determina la intensidad máxima en la punta de la fibra. Las más pequeñas poseen intensidad más elevada. Este efecto se corrige por la divergencia del rayo a la salida de la fibra, así a una corta distancia una fibra de 350 micrones es igual que una de 600 micrones (4). Las fibras más finas son más frágiles y se utilizan para uréter y riñón.

Los láseres pulsados (Holmiun, Thulium) son excelentes para la fragmentación de los cálculos, estenosis uretrales, esclerosis de cuello, pequeños tumores de la vía urinaria (5 y 7). Cada pulsación produce una onda de rápida expansión y contracción creando una burbuja de vapor (Moses effect). La burbuja dinámica (pico de potencia) es la que actúa en el tejido blando y lo arranca aparte. El diámetro de la misma es de escasos milímetros dependiendo de la energía láser pulsada. La vida media de la burbuja es igual a la duración de la onda láser pulsada, aproximadamente $500 \mathrm{~ms}$; este tiempo, es demasiado corto para la imagen videoscópica de control, explicando el por qué la burbuja es invisible $(5,6)$.

El corte con el modo continuo es preciso. La onda continua no provoca la formación de la burbuja de vapor y la calidad del corte es superior, sin alterar la visión del campo operatorio, siendo visualizado especialmente en cirugía laparoscópica. La absorción del láser Revolix $\circledast$ es 2.5 veces superior a la del láser Holmiun.

En las próstatas muy fibrosas donde no hay suficiente hemoglobina, los láseres que actúan solamente donde ella existe como por ejemplo el KTP, la vaporización se reduce y el efecto térmico aumenta provocando necrosis tisular; esto origina disuria y síndrome irritativo prolongado (7).

El láser Revolix® elimina el tejido prostático con una media superior a $1.5 \mathrm{gr} / \mathrm{min}$. y coagula vasos de hasta $1.6 \mathrm{mms}$. El efecto de la onda láser por debajo del corte es de $0.2 \mathrm{mms}(8,9$ y10).

La HBP podemos tratarla con el láser Revolix ${ }^{\circledR}$ con dos tipos diferentes de fibras: La fibra frontal (rigifib) se utiliza para la vaporesección, que corta y a la vez vaporiza. Esto es ideal para tratar todo tipo de próstatas, aunque en nuestra experiencia la utilizamos para las mayores de 35 grs. Los cortes no se modifican en los tejidos fibrosos. Esta fibra frontal es multiuso, y se pueden realizar hasta 40 tratamientos con una sola, lo que equivale aproximadamente a un coste de 20 euros por paciente. Esta es una de las diferencias importantes con otros equipos de láser utilizados para el tratamiento de la HBP.

Los pacientes fueron evaluados con examen físico que incluía tacto rectal, estudio analíitico de laboratorio con PSA, ecografia renal y vesicoprostática (medición de la próstata en grs). Los estudios con flujometría (Qmax), el cuestionario de IPSS y la medición del residuo postmiccional, se realizaron en el preoperatorio, después de retirada la sonda vesical y a los 3 y 12 meses (Tabla II).

\section{Técnica quirúrgica de la cirugía prostática con láser Revolix $\otimes_{0}$}

Utilizamos un resectoscopio $26 \mathrm{Ch}$ de Wolf $\mathrm{C}$ flujo continuo. La óptica es de $30^{\circ}$ con un elemento de

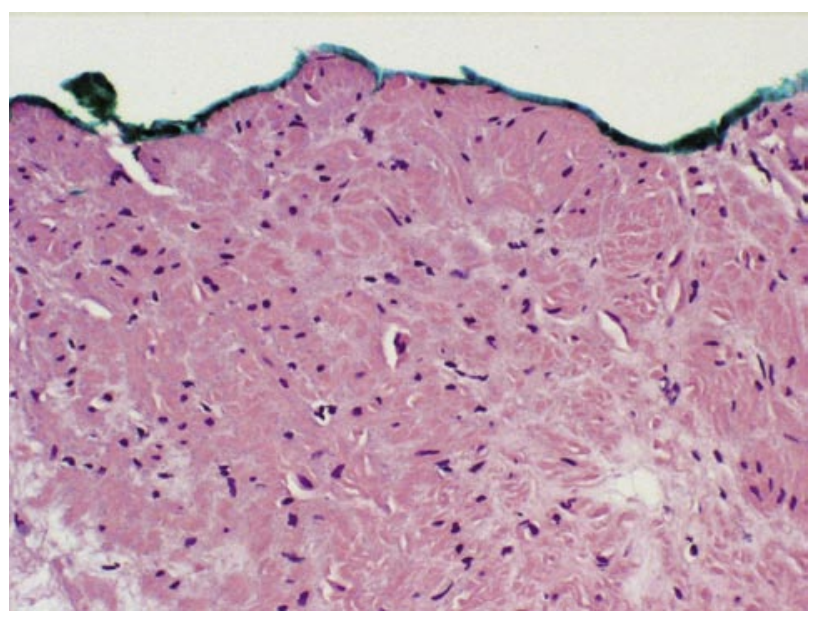

FIGURA 3. Se puede observar en la imagen que la afectación de tejido prostático es mínima

Tinción Hematoxilina Eosina $20 x$.

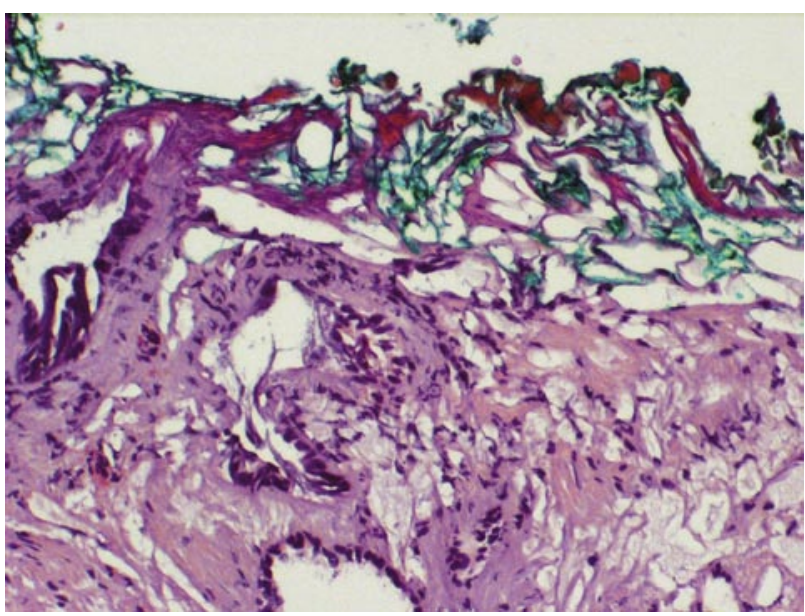

FIGURA 4. Tinción Hematoxilina-Eosina $100 x$. 
trabajo que pemite movilizar la fibra frontal como en una RTU. Con la fibra de vaporesección se hace una técnica que denominamos en "lavaparabrisas", es decir, se realiza lateralmente un corte de ida y vuelta con la fibra en forma de arco, sin tener un contacto demasiado profundo con el tejido; de esta manera, el tejido prostático se va cortando y a la vez vaporizando de atrás hacia delante hasta lograr desprenderlo en la vejiga. Es muy importante tener un buen flujo de irrigación salina que ayuda a movilizar el fragmento que estamos vaporesecando.

Se inicia el tratamiento con el lóbulo medio realizando dos surcos a las 5 y 7 del horario cistoscópico. La sección se hace desde el cuello vesical hasta llegar a la altura del veru montanum. A partir de ahí, el resto de tejido prostático que queda entre los dos surcos se va vaporesecando hasta llegar al cuello vesical y se desprenda en la vejiga. Los fragmentos no deben ser voluminosos, de esta forma podemos extraerlos con el evacuador de Ellick. En algunos casos de próstatas voluminosas, hemos tenido que utilizar el resector bipolar para disminuir el volumen de los fragmentos en la vejiga. No utilizamos morcelador.

Los lóbulos laterales pueden resecarse realizando un surco a la 1 del horario cistoscópico, hasta aproximarse a la cápsula y desde ahi continuar con la técnica en "lavaparabrisas". En algunos casos preferimos iniciar el corte a las 5 y 7 del horario cistoscópico, a cada lado del veru montanum e ir ascendiendo hasta la parte media del lóbulo lateral. Otros autores han descrito la técnica tangerine (mandarina). Esta técnica consiste en que se van realizando surcos y posteriormente se vaporeseca desde atrás hacia el cuello consiguiendo desprender los fragmentos en la vejiga. Cuando tratan el lóbulo medio realizan un surco a las 5,6 y 7 horas hasta el veru. Con el lóbulo lateral izquierdo se efectúan surcos a la 1, 3 y 5 como si se formaran gajos de una mandarina. En el lóbulo contralateral se realizan exactamente surcos a las 7,9 y 11 horas $(2,11)$.

La fibra lateral (sidefib) se usa para vaporización. La técnica es semejante a la utilizada con el láser KTP (3).

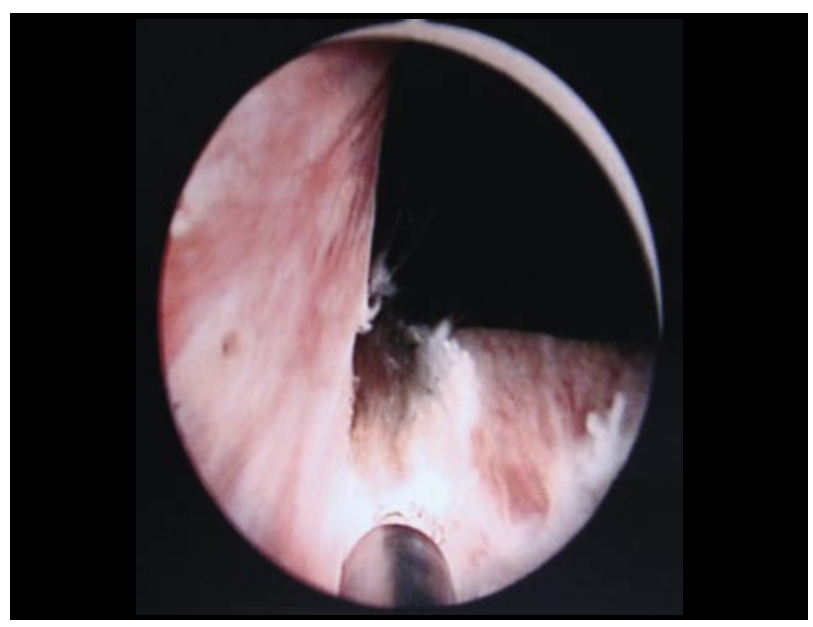

FIGURA 5. Imagen endoscópica donde se puede visualizar el surco realizado con vaporesección a las 7 horas del horario cistoscópico.
Esta fibra se puede utilizar 2 o 3 veces, pues depende del tamaño de la próstata. El coste del proceso es aproximadamente 600 euros. Generalmente se usa para tratamiento de los adenomas menores de 35 gramos, consiguiendo resultados muy buenos.

En todos los pacientes con HBP sometidos a tratamiento mediante el láser Revolix ${ }^{\circledR}$, se pueden utilizar ambas fibras, frontal y lateral, para combinar la vaporesección con la vaporización, consiguiendo con ello unos excelentes resultados endoscópicos como se puede apreciar en las imágenes endoscópicas (Figuras 5, 6, 7 y 8). (Tabla I).

\section{RESULTADOS}

Entre noviembre 2004 y marzo 2008, se trataron 200 pacientes con HBP mediante el láser Revolix® de 70 vatios. Se desestimó realizar tratamientos en pacientes con cáncer de próstata y en aquellos pacientes que estaban diagnosticados de vejiga neurogénica. No es una contraindicación de realizar tratamiento con láser Revolix $®$ en aquellos pacientes que estén anticoagulados.

Tres pacientes tenían como patología asociada un tumor superficial de vejiga, el cual fue tratado en el mismo tiempo operatorio con el láser Revolix $®$. Dos pacientes con litiasis múltiples de vejiga asociada se trataron con éxito mediante la percufibra y la fibra frontal, como hace Fried et al (12). Tres pacientes presentaban estenosis de uretra bulbar menores de $1 \mathrm{~cm}$ de longitud, la cuales fueron tratadas con láser a modo continuo con una potencia de 15 vatios, previo a la vaporeseccion de la HBP.

La edad promedio de los pacientes tratados fue de 62 años (rango, 45-89). Había 21 pacientes portadores de sonda vesical permanente. El tamaño promedio de la próstata oscilo entre 20 y 120 grs (promedio $45 \mathrm{grs}$ ). 12 pacientes estaban anticoagulados (pacientes de alto riesgo). El tiempo operatorio estuvo entre 25 y 140 minutos. Ningún paciente requirió transfusión sanguinea post opera-

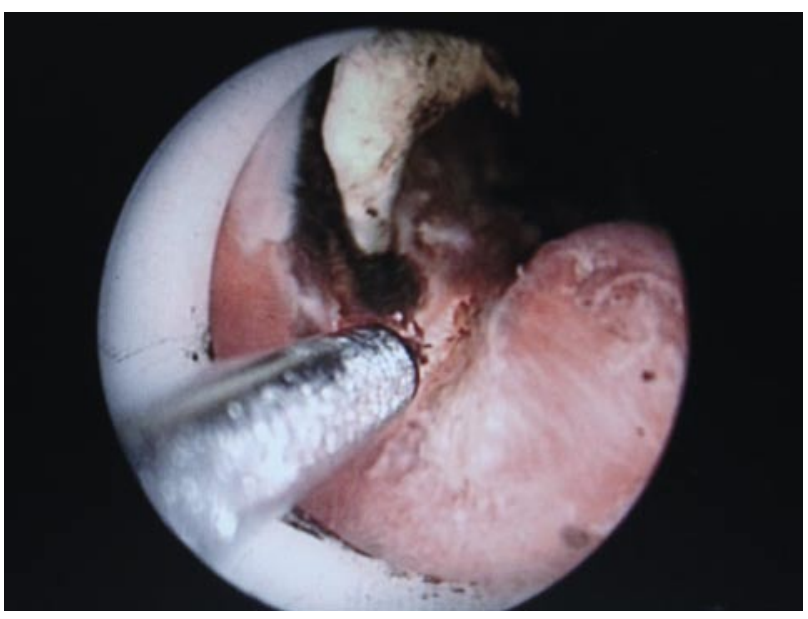

FIGURA 6. Imagen endoscópica del surco realizado con vaporesección a las 7 horas. Obsérvese el veru montanum cercano a la fibra de láser. 
TABLA I. DIFERENCIAS EXISTENTES ENTRE LA VAPORESECCIÓN Y LA VAPORIZACIÓN CON LÁSER REVOLIX®.

\begin{tabular}{|l|c|c|}
\hline & Vaporización & Vaporesección \\
\hline Próstata & $<35 \mathrm{grs}$ & $>35 \mathrm{y}<120 \mathrm{grs}$ \\
\hline Muestras AP & No & $\mathrm{Si}$ \\
\hline Costo & Hasta 3 usos & Múltiples usos \\
\hline Velocidad & Similar KTP & Igual al Holmiun \\
\hline
\end{tabular}

toria. El promedio de permanencia de la sonda vesical tras la operación fue de 16 horas (rango, 12-72 horas).

Los 101 pacientes, con próstatas mayores de 35 gramos y menores de 120, fueron tratados mediante la fibra frontal, practicando una vaporesección. La extracción de las muestras se hace mediante evacuador de Ellick ya que no utilizamos morcelador para extraer los fragmentos. En 6 casos utilizamos el resector bipolar para la extracción de fragmentos grandes que no pasaban por la vaina del resectoscopio (próstatas voluminosas). Los fragmentos enviados para estudio anatomopatológico no evidenciaron cambios histológicos significativos (Figura 2). 99 pacientes se trataron con la fibra lateral (vaporización), que fueron aquellos con próstatas menores de 35 grs.

Los urólogos con experiencia en resecciones transuretrales no tienen dificultad con estas técnicas endoscópi-

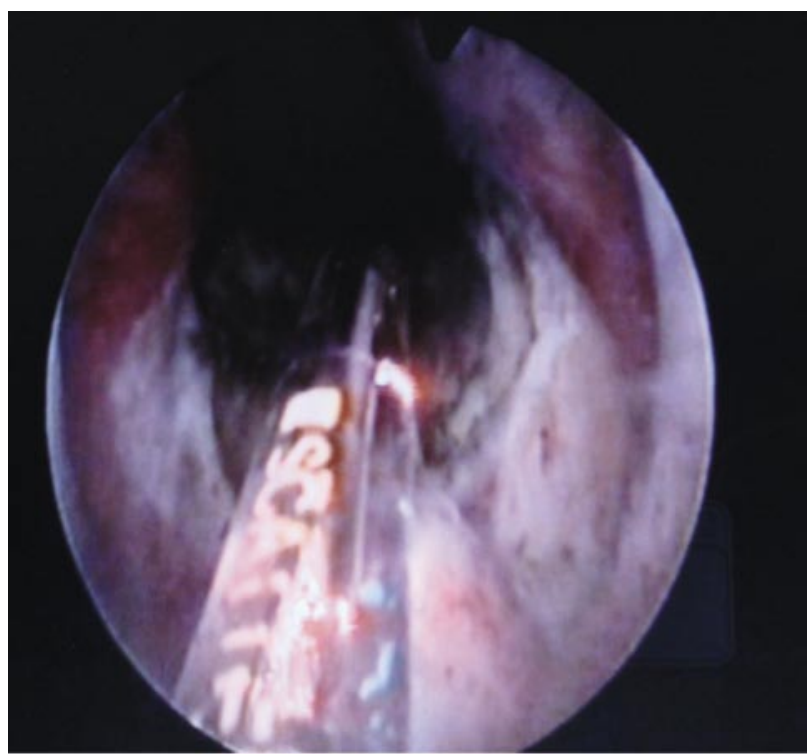

FIGURA 7. Imagen endoscópica de la fibra sidefib, realizando vaporización del tejido prostático. cas con láser, suponiendo una curva de aprendizaje muy corta. Ningún paciente necesitó tratamiento adicional por complicaciones, tales como la esclerosis de cuello vesical - la estenosis de uretra. 7 pacientes fueron reintervenidos por una insuficiente vaporización del tejido prostático, que coincidió con la curva de aprendizaje de los primeros casos. En 5 casos se produjo sangrado durante el tratamiento y en 10 casos existió sangrado post-tratamiento, pero en ningún caso fue preciso realizar transfusión sanguínea. 4 pacientes presentaron retención urinaria después de retirada la sonda. Un 6 \% de los pacientes presentaron fenómenos irritativos durante varios dias tras retirada de la sonda vesical (entre 1 y 3 semanas), principalmente en aquellos pacientes tratados con vaporización.

Los resultados funcionales obtenidos con el tratamiento de la HBP mediante láser Revolix ${ }^{\circledR}$ son comparables a los obtenidos con la RTU, PVP y HoLEP. Las diferencias del laser Revolix ${ }^{\circledR}$ con respecto a la RTU son la ausencia del síndrome de reabsorción de líquidos de irrigación, no precisar lavados vesicales en el post-operatorio, no descenso significativo en la tasa de hemoglobina, menor tiempo de sonda vesical (menos de $24 \mathrm{hs}$ ) y corto período de hospitalización $(13,14,15)$. La posibilidad de utilizar dos tipos de fibras con distintas funciones lo diferencia de otros laseres. La fibra lateral (vaporización) la hemos utilizado para tratar las próstatas menores de $35 \mathrm{grs}$ y la fibra frontal (vaporesección) las superiores a esta cifra. La onda continua del laser Revolix ${ }^{\circledR}$ tiene una absorción mucho mas importante que el laser Holmiun (onda pulsada), no produce burbujas (efecto Moser) (5), se logra un corte más preciso y tiene una curva menor de aprendizaje $(3,9$ y 16$)$, además existe menor riesgo de perforar la cápsula prostática, lesionar la mucosa vesical y el meato ureteral (3). Con respecto al laser KTP, el láser Revolix® ofrece la posibilidad de utilizar la rigidfiber hasta 40 veces lo que significa un coste de 20 euros por tratamiento (la fibra del KTP tiene un coste

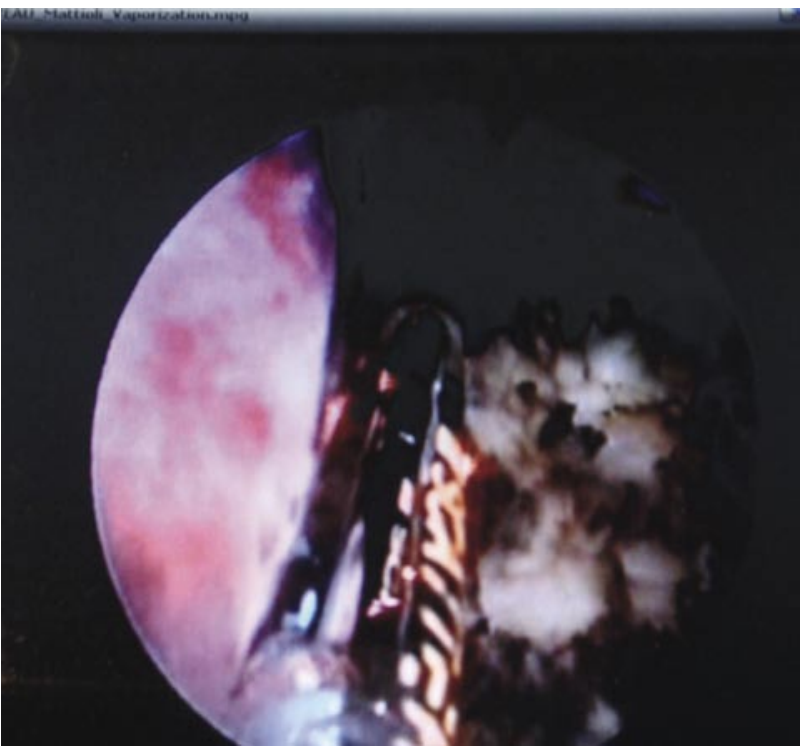

FIGURA 8. Imagen endoscópica donde se muestra la fibra sidefid y el tejido prostático que está siendo vaporizado. 
TABLA II. RESULTADOS FUNCIONALES PRE Y POSTOPERATORIOS EN LOS PACIENTES TRATADOS CON LÁSER REVOLIX®.

\begin{tabular}{|l|c|c|c|c|}
\hline & Pre-operatorio & Post-operatorio & 3 meses & 12 meses \\
\hline Sintomatología urinaria (Score AUA) & 21.5 & 7.8 & 7.5 & 6.9 \\
\hline Q-max (ml/sec) & $0-7.8$ & 21.2 & 21.6 & 22.6 \\
\hline Residuo postmiccional (ml) & 112.7 & 14.6 & 12.8 & 12.5 \\
\hline
\end{tabular}

aproximado por tratamiento de 1200 euros, ya que no es reutilizable. En próstatas voluminosas con el laser KTP es necesario utilizar mas de una fibra y eso encarece aun más el tratamiento (16). La vaporesección permite extraer fragmentos para biopsia y no así cuando se hace vaporización con el KTP. El agua es el target cromóforo del laser

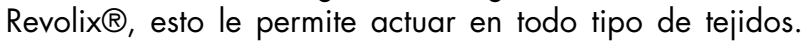
La oxihemoglobina es el target cromóforo del laser KTP, de esta manera en las próstatas fibrosas, su acción se ve disminuida provocando un aumento de la temperatura en estos tejidos hasta la necrosis tisular.

En la Tabla II se muestran los resultados de los pacientes tratados con láser Revolix®. Observamos una importante disminución de los síntomas de más del 50\% del IPSS, un aumento significativo de los valores de la flujometría y un descenso marcado del residuo post miccional.

\section{CONCLUSIONES}

En nuestra experiencia, el láser Revolix ${ }^{\circledR}$ ha demostrado ser muy seguro, de fácil instalación, con bajo costo operativo y con una curva de aprendizaje muy corta. Su efectividad se puede poner más de manifiesto empleando la vaporesección en las próstatas voluminosas y la vaporización en las próstatas más pequeñas (menores de 35 grs). La reducción del tejido prostático es inmediata con un ligero disconfort post operatorio y un bajo índice de complicaciones.

\section{BIBLIOGRAFÍA y LECTURAS RECOMENDADAS ("lectura de interés $\mathrm{y}^{* *}$ lectura fundamental)}

1. MATSUOKA, K.; LIDA, S.; TOMIYASU, K. y cols.: "Transurethral holmiun laser resection of the prostate". J. Urol., 163: 515, 2000.

**2. XIA, S.J.; ZHU, J. y cols.: "The treatment of benign prostatic hyperplasia by means of transurethral holmiun laser enucleation". Zhonghus Nan Ke Xue, 9: 257, 2003.

3. HEINRICH, E.; SCHIEFELBEIN, F; SCHOEN, G.: "Technique and short-term outcome of green light la- ser (KTP,80 W) vaporization of the prostate". European Urology, 52: 1632, 2007.

**4. NASIF, O.A.; TEICHMAN, J.M.; GLICKMAN, R.D. y cols.: "Review of laser fibers a practical guide for urologists”. J. Endourol., 18: 818, 2004.

*5. YARBOROUGH, J.M.: "Taking the confusion out of matching medical lasers". Lasers and Laser Systems. Medical Lasers. The photonics design and application handbook, 287, 1996.

6. KUNTZ, R.M.: "Lasers treatment of bening prostatic hyperplasia". World J. Urol., 2007.

**7. TEICHMANN, H.O.; HERMANN, T.R.; BACH, T.: "Technical aspects of lasers in urology". World J. Urol., 25: 221, 2007.

8. BACH, T.; HERMANN, T.R.; CELLARIUS, C. y cols.: "EAU annual meeting, session abstract" 2007.

**9. FRIED, N.M.: "High-power laser vaporization of the canine prostate using a $10 \mathrm{~W}$ thulium fiber laser at 1.91 $\mu$ m”. Laser Surg. Med., 36: 52, 2005.

10. TE, A.E.; MALLOY, T.R.; STEIN, B.S. y cols.: "Photoselective vaporization of the prostate for the treatment of benign prostatic hyperplasia: 12 month results from the first United States multicenter prospective trial". J. Urol., 172: 1404, 2004.

**11. XIA, S.J.; ZHANG, Y.N.; LU, J. y cols.: "Thulium laser resection of prostate tangerine technique in treatment of benign prostate hyperplasia". Zhonghus Nan Ke Xue, 85: 3225, 2005.

*12. FRIED, N.M.: “Thulium fiber laser lithotripsy: An n vitro analysis of stone fragmentation using a modulated 110-Watt thulium fiber laser at 1.94 micron". Laser Surg. Med., 37: 53, 2005.

13. SHINGLETON, W.B.; FARABAUGH, P.; MAY, W.: "Three years follow-up laser prostatectomy versus transurethral resection of the prostatic hyperplasia". Urology, 48: 575, 1996.

**14. XIA, S.J.; ZHUI, J.; XIAO, W.S. y cols.: "Thulium laser versus standard transurethral resection of the prostate: A randomized prospective trial". Eur. Urol., 53: 382, 2008.

*15. FRIED, N.M.; MURRAY, K.E.: "High thulium fiber laser ablation of urinary tissues at $1.94 \mathrm{~mm}$ ". J. Endourol., 19: 25, 2005.

16. KUNTZ, R.M.: "Current role of lasers in the treatment of benign prostatic hyperplasia (BPH)". Eur. Urol., 49: 961, 2006. 